\title{
ENHANCEMENT OF TURBULENT VISCOSITY BY GLOBAL MAGNETIC FIELDS IN ACCRETION DISKS
}

\author{
YASUSHI NAKAO \\ Department of Astronomy, Faculty of Science, Kyoto University \\ Sakyo-ku, Kyoto, 606-01, Japan
}

\section{Summary}

A model of magnetohydrodynamic (MHD) turbulence in accretion disks with global magnetic fields is constructed using a second-order closure modeling of turbulence. The transport equations of the Reynolds stress tensor, the Maxwell stress tensor, and the cross-helicity tensor (the correlation of velocity fluctuation and magnetic fluctuation) are closed by second-order quantities using a two-scale direct interaction approximation (TSDIA). The quantities appearing these equations are considered to be those averaged in the vertical direction of the disks. The turbulence is assumed to be stationary. We are interested only in the effects of the global magnetic fields on the turbulence in the disks, i.e., no dynamo processes are considered, and the global magnetic fields are supposed to be embedded in the disk $a$ priori.

The results show that the presence of a radial global field enhances the turbulent viscosity (and the so-called $\alpha$-parameter value) in the disk. This suggests that the radial global magnetic field has something to do with advection-dominated disks, in which a rather large viscosity is needed. In advection-dominated disks, the advective motion of the gas is likely to produce a radial global field. On the other hand, both toroidal and vertical global fields diminish the strength of the turbulence. Therefore, the strength of the turbulence in the accretion disk is determined by competition between the above-mentioned two opposite effects of the global fields. Our results show that the enhancement of the turbulence due to a radial field is more effective than the suppression of the turbulence due to a toroidal (or a vertical) field. See Nakao (1997) (Publ. Astron. Soc. Japan 49, in press) for the details. 